\title{
Generalization of coupled $S$-parameter calculation to compute beam impedances in particle accelerators
}

\author{
Thomas Flisgen * \\ Ferdinand-Braun-Institut, Leibniz-Institut für Höchstfrequenztechnik, \\ Gustav-Kirchhoff-Straße 4, 12489 Berlin, Germany \\ Erion Gjonaj \\ Institute for Accelerator Science and Electromagnetic Fields, Technische Universität \\ Darmstadt, Schloßgartenstraße 8, 64289 Darmstadt, Germany \\ Hans-Walter Glock and Andranik Tsakanian \\ Helmholtz-Zentrum Berlin, Albert-Einstein-Straße 15, 12489 Berlin, Germany
}

(Received 11 October 2019; accepted 21 January 2020; published 11 March 2020)

\begin{abstract}
In this article, a decomposition approach for the computation of beam coupling impedances is proposed. This approach can account for the mutual electromagnetic coupling in long accelerator structures consisting of several consecutive segments. The method is based on the description of the individual segments using a multimodal network matrix formulation in which the charged particle beam is considered as an additional port. Then, the generalized multimodal network matrices of all segments are combined to a multimodal network matrix of the complete structure. The beam coupling impedance as well as the scattering parameters of the full structure are recovered as particular matrix elements in this multimodal network matrix. The new method generalizes Coupled $S$-Parameter Calculation (CSC) introduced in earlier work such that charged particle beams are considered. Consequently, the introduced scheme is referred to as $\mathrm{CSC}^{\mathrm{BEAM}}$. Application examples for realistic accelerator components such as the simulation of a full TESLA 1.3 GHz-cavity of the European XFEL are provided. These simulations demonstrate the high accuracy and numerical performance of the proposed method.
\end{abstract}

DOI: 10.1103/PhysRevAccelBeams.23.034601

\section{INTRODUCTION}

The characterization of the interaction between charged particle beams and vacuum chambers of accelerator components is a common task in accelerator physics. Bunches of charged particles traversing vacuum chambers of accelerators interact with these chambers and excite electromagnetic fields. These fields are referred to as wakefields. The wakefields can lead to unwanted heating of accelerator parts or may interact with subsequent bunches deflecting the charged particles from their ideal trajectories. This interaction can result in instabilities during the operation of the machine and in the worst case in the total loss of the beam [1]. Wake potentials are typically employed to characterize the aforementioned processes in time domain whereas beam coupling impedances are the counterpart for

\footnotetext{
*thomas.flisgen@fbh-berlin.de

Published by the American Physical Society under the terms of the Creative Commons Attribution 4.0 International license. Further distribution of this work must maintain attribution to the author(s) and the published article's title, journal citation, and DOI.
}

a description in frequency domain. The concept of coupling impedances was first proposed by Vaccaro [2] in the sixties of the last century in combination with the concept of stability diagrams to systematically study instabilities in circular accelerators. The reader is referred to [3] for a historical review of these closely related concepts.

Numerical simulation is a well established tool for the determination of beam coupling impedances. The typical procedure consists of subdividing the accelerator line into a large number of subsegments. Then, the individual coupling impedances of each segment are determined either by means of analytical estimations for segments with simple geometries or by means of numerical codes such as ECHO [4], PBCI [5], GDFIDL [6], or CST STUDIO SUITE® [7] for sophisticated geometries. Alternatively, coupling impedances of segments can be acquired by bench measurements. In a final step, the individual impedances of the segments are added up to obtain a global impedance model of the accelerator. Figure 4.1 in [8] presents a functional diagram of this approach. The described method delivers accurate results if the electromagnetic fields in the respective segments are sufficiently decoupled. However, the results are inaccurate if the segments are electromagnetically coupled, 
for instance by the high-pass characteristic of the waveguidelike beam pipes connecting adjacent segments. In this case, resonant modes with field energy distributed along various segments can exist. These modes can significantly contribute to the global impedance spectrum of the machine.

On the other hand, the numerical impedance computation for large accelerator structures consisting of electromagnetically coupled cavities using conventional simulation tools is extremely cumbersome. Most of these tools are based on the direct simulation of the wakefields in the time domain for the complete structure. Hereby, a discretization technique based on the finite integration technique (FIT) [9] is usually applied. This approach, however, leads to inherently long simulation times due to the long wakefield transients within the structure. This is particularly the case for the short electron bunches that are operated in new generation x-ray sources such as E-XFEL or LCLS II, where the "catch-up" distance of the wakefields is by orders of magnitude longer than the bunch length [10]. Furthermore, the size of the discrete problem becomes often prohibitively large, even when a moving window technique combined with massive parallelization is employed [11]. This is due to the very fine Cartesian-type meshes that are required by the FIT discretization to resolve the bunch current as well as the small geometrical details of the structure.

The aim of this article is the introduction of an approach which is able to compute the beam coupling impedance of large and geometrically complex accelerator structures based on the simulation of smaller subsegments that can be performed very efficiently using moderate computational resources. Figure 1 depicts the main idea of the method, i.e., the decomposition of a beamline section into different elements. The new approach builds upon the previously introduced method of Coupled $S$-Parameter Calculation (CSC) [12-17]. CSC delivers multimodal scattering matrices of complex geometries based on multimodal scattering matrices of segments of the geometry in combination with topology information. In this article, the
CSC formulation is generalized so that the coupling of the beam to the cavity fields is accounted for. Since the introduced scheme is a generalization of CSC, it is referred to as $\mathrm{CSC}^{\mathrm{BEAM}}$. The first attempt to include the charged particle beam in CSC is described in [18] which is focused on port signals in time domain and not on impedances.

The article is organized as follows. In Sec. II, the commonly known concept of scattering matrices is generalized so that charged particle beams are considered as an additional port. Thereafter, the numerical determination of these generalized matrices for individual segments using a finite element formulation is presented. In Sec. II C, relevant aspects of the $\mathrm{CSC}^{\mathrm{BEAM}}$ approach are introduced. This includes the decomposition of the structure of interest into segments, the concatenation of the generalized beam coupling matrices of the respective segments, and the reconstruction of global field distributions. In Sec. III, the application of $\mathrm{CSC}^{\mathrm{BEAM}}$ for two typical accelerator cavity structures is demonstrated. In particular, the impedance calculation for a full $1.3 \mathrm{GHz}$ TESLA nine-cell cavity of the European XFEL is presented. Finally, Sec. IV provides a summary and conclusions.

\section{THEORY}

\section{A. Generalization of scattering matrices}

In the following, the problem of electromagnetic wakefields in accelerators caused by a relativistic charged particle beam in the longitudinal $z$-direction is considered. It is assumed that the rigid-beam approximation applies so that the coupling between the field equations and the particle equations of motions can be neglected [19]. For CSC the spectral properties of every segment are described by their multimodal frequency-dependent scattering matrices $\underline{\mathbf{S}} \in \mathbb{C}^{N \times N}$. This requires the segments to consist of linear materials so that the principle of superposition applies. To account for the interaction of a structure with a charged particle beam, the latter is considered as an additional port in the scattering matrix. This idea of treating the particle beam on the same footing

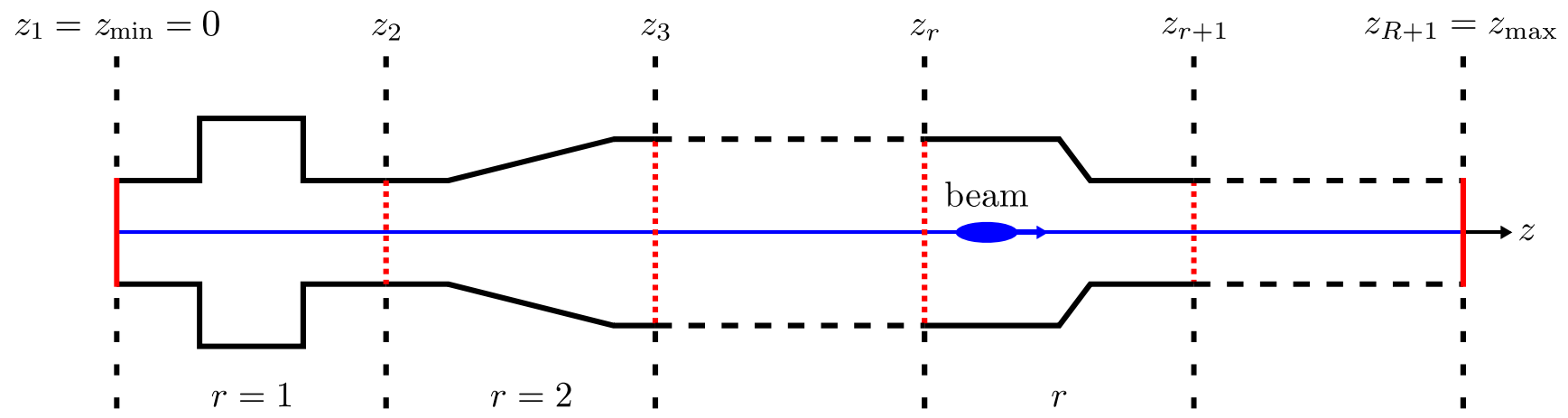

FIG. 1. Example of the decomposition of a beamline section into $R$ individual segments. The decomposition planes are marked using red dotted lines. Every segment is indexed by $r$. The beam current $\underline{\mathbf{J}}\left(x_{\mathrm{b}}, y_{\mathrm{b}}, z\right)$ is indicated as blue line. The beam enters the structure at $z_{\min }=z_{1}=0$ (left red solid line) and leaves the structure at $z_{\max }=z_{R+1}$ (right red solid line). 
with the waveguide modes as an additional discrete port characterized by currentlike and voltagelike quantities is originally proposed in [20].

Extending the scattering matrix by one row and one column to accommodate the beam excitation as an additional port yields

$$
\underbrace{\left(\begin{array}{ll}
\underline{\mathbf{S}} & \underline{\mathbf{k}} \\
\underline{\mathbf{h}} & \underline{z_{\mathrm{b}}}
\end{array}\right)}_{\tilde{\mathbf{s}}} \underbrace{\left(\begin{array}{l}
\underline{\mathbf{a}} \\
\underline{i}
\end{array}\right)}_{\underline{\tilde{\mathbf{a}}}}=\underbrace{\left(\begin{array}{l}
\underline{\mathbf{b}} \\
\underline{v}
\end{array}\right)}_{\underline{\tilde{\mathbf{b}}}},
$$

where underlined symbols refer to complex-valued quantities which are sampled at a given frequency. The vectors $\underline{\mathbf{a}} \in \mathbb{C}^{N \times 1}$ and $\underline{\mathbf{b}} \in \mathbb{C}^{N \times 1}$ comprise the incident and scattered wave amplitudes corresponding to all possible (in total $N$ ) modal excitations at the waveguide boundaries of the structure. When $M$ waveguide modes are considered at the waveguide boundary located at $z_{\min }$ (see Fig. 1), the incident and scattered wave amplitudes are related to tangential electric and magnetic fields in the frequency domain as follows:

$$
\begin{aligned}
\underline{\mathbf{E}}_{\mathrm{t}}\left(x, y, z_{\min }\right)= & \sum_{m=1}^{M} \mathbf{e}_{m}(x, y) \sqrt{\underline{Z}_{m}} \\
& \left(\underline{a}_{m} \mathrm{e}^{-j \underline{k}_{z, m} z_{\min }}+\underline{b}_{m} \mathrm{e}^{j \underline{k}_{z, m} z_{\min }}\right)
\end{aligned}
$$

and

$$
\begin{aligned}
\underline{\mathbf{H}}_{\mathrm{t}}\left(x, y, z_{\min }\right)= & \sum_{m=1}^{M} \frac{\mathbf{n}_{z} \times \mathbf{e}_{m}(x, y)}{\sqrt{\underline{Z}_{m}}} \\
& \left(-\underline{a}_{m} \mathrm{e}^{-j \underline{k}_{z, m} z_{\min }}+\underline{b}_{m} \mathrm{e}^{j \underline{k}_{z, m} z_{\min }}\right),
\end{aligned}
$$

where $\mathbf{e}_{m}(x, y)$ are the modal functions on the port plane, $\underline{Z}_{m}$ the frequency-dependent wave impedances, $\underline{k}_{z, m}$ the frequency-dependent propagation constants, and $\underline{a}_{m}$ and $\underline{b}_{m}$ are the incident and scattered wave amplitudes of the $m$ th waveguide mode, respectively. The normal vector pointing inside the structure in the $z$-direction is denoted by $\mathbf{n}_{z}$. Furthermore, given a known incident field described by the coefficients $\underline{a}_{m}$ and assuming mode orthogonality, the scattered wave amplitudes $\underline{b}_{m}$ can be computed directly from the electromagnetic field (2) and (3).

For a point charge moving in the $z$-direction with constant velocity $\beta c_{0}$ and transversal offsets $x_{\mathrm{b}}$ and $y_{\mathrm{b}}$, the current density in the frequency domain is related to the beam current $\underline{i}$ in (1) by

$$
\underline{J}_{z}(x, y, z)=\underline{i} \delta\left(x-x_{\mathrm{b}}\right) \delta\left(y-y_{\mathrm{b}}\right) \exp \left(-\frac{j \omega z}{\beta c_{0}}\right),
$$

where $\delta$ is the Dirac delta function, $j$ the complex unit, $\omega$ the angular frequency, $\beta<1$, and $c_{0}$ the speed of light in vacuum. It is assumed throughout the article that the port plane where the beam enters the structure is located at $z_{1}=z_{\min }=0$ (cf. Fig. 1).

The voltage $\underline{v}$ in (1) denotes the voltage induced on the point charge and is defined as

$$
\underline{v}=\int_{z_{\min }}^{z_{\max }} \underline{E}_{z}\left(x_{\mathrm{b}}, y_{\mathrm{b}}, z\right) \exp \left(\frac{j \omega z}{\beta c_{0}}\right) \mathrm{d} z,
$$

where $z_{\max }$ is the position of the port plane of the outgoing beam pipe. Furthermore, $\underline{E}_{z}\left(x_{\mathrm{b}}, y_{\mathrm{b}}, z\right)$ denotes the longitudinal electric field strength along the point charge path.

In addition to the commonly known multimodal scattering matrix $\underline{\mathbf{S}}$, the generalized matrix $\underline{\tilde{\mathbf{S}}} \in \mathbb{C}^{(N+1) \times(N+1)}$ comprises the row vector $\underline{\mathbf{h}} \in \mathbb{C}^{1 \times N}$, the column vector $\underline{\mathbf{k}} \in \mathbb{C}^{N \times 1}$, and the scalar coefficient $\underline{z}_{\mathrm{b}} \in \mathbb{C}^{1 \times 1}$. The vector $\underline{\mathbf{h}}$ describes the contribution of incident waveguide waves $\underline{\mathbf{a}}$ to the voltage $\underline{v}$. The coefficients of $\underline{\mathbf{h}}$ can be determined by exciting the structure one-by-one with all its $N$ waveguide modes and then computing for each excitation the induced beam voltage (5). The vector $\underline{\mathbf{k}}$ describes the coupling of the beam to the $N$ waveguide modes. The coefficients of $\underline{\mathbf{k}}$ correspond to the scattered wave amplitudes $\underline{\mathbf{b}}$ at the waveguide ports when the system is solely excited by the beam current. The transfer functions in $\underline{\mathbf{k}}$ are particularly relevant for the estimation of beam signals for beam position monitors or for the power at higher-order mode ports as discussed in [21,22]. The scalar $\underline{z}_{\mathrm{b}}$ describes the relationship between the beam current $\underline{i}$ and the voltage $\underline{v}$ seen by the beam when no waveguide mode is excited. As a result of (4) and (5), this coefficient is the commonly known longitudinal impedance [23]. The quantities $\underline{\mathbf{h}}, \underline{\mathbf{k}}$ and $\underline{z}_{\mathrm{b}}$ do depend on the transversal offsets $x_{\mathrm{b}}$ and $y_{\mathrm{b}}$.

It is obvious from the definition (1) that the generalized matrix relates quantities of different kind, i.e., incident waves, scattered waves, a current, and a voltage. Therefore, the formulation may be considered as a hybrid representation, where the scattering matrix approach for the waveguide modal amplitudes is combined with an impedance representation for the particle beam. Note that the unit of the scattering matrix $\underline{\mathbf{S}}$ is dimensionless, the dimension of $\underline{\mathbf{h}}$ and $\underline{\mathbf{k}}$ is $\sqrt{\Omega}$, and that of $\underline{z}_{\mathrm{b}}$ is $\Omega$.

\section{B. Finite element impedance solver}

In order to determine the generalized beam coupling matrices (1), the solution of Maxwell's equations for each considered frequency is needed. Apart from the calculation of the conventional scattering matrix block $\underline{\mathbf{S}}$ in (1), the procedure requires the estimation of the induced voltage on the beam axis (5) as well as the modal scattering coefficients $\underline{\mathbf{b}}$, generated by the beam.

For the solution of the field problem, a high-order finite element approach in the frequency domain is adopted. Given a discretization of the computational domain $\boldsymbol{\Omega}$, 
the electric field strength is approximated by a linear combination of vector basis functions on the mesh. The appropriate approximation space is the tangentially continuous $H($ curl $)$-space defined as, $H($ curl $)=\left\{\boldsymbol{\phi}_{h} \in \mathbf{L}^{2}\right.$ : curl $\left.\phi_{h} \in \mathbf{L}^{2}\right\}$. High-order hierarchical $H($ curl)-basis functions for various polygonal type meshes are given, e.g., in [24]. Then, following the standard Galerkin procedure [25], the weak form of the frequency-domain problem reads

$$
\begin{aligned}
& \iiint_{\boldsymbol{\Omega}} \mu_{\mathrm{r}}^{-1} \nabla \times \underline{\mathbf{E}} \cdot \nabla \times \boldsymbol{\phi}_{h} \mathrm{~d} V-k_{0}^{2} \iiint_{\boldsymbol{\Omega}} \varepsilon_{\mathrm{r}} \underline{\mathbf{E}} \cdot \boldsymbol{\phi}_{h} \mathrm{~d} V \\
& \quad=-j k_{0} Z_{0} \iiint_{\boldsymbol{\Omega}} \underline{\mathbf{J}} \cdot \boldsymbol{\phi}_{h} \mathrm{~d} V-\oiint_{\partial \boldsymbol{\Omega}} \mu_{\mathrm{r}}^{-1} \boldsymbol{\phi}_{h} \cdot[\mathbf{n} \times \nabla \times \underline{\mathbf{E}}] \mathrm{d} A,
\end{aligned}
$$

$\forall \boldsymbol{\phi}_{h}=\boldsymbol{\phi}_{h}(x, y, z) \in H($ curl $)$, where $\underline{\mathbf{E}}=\underline{\mathbf{E}}(x, y, z)$ is the electric field strength, $\underline{\mathbf{J}}=\mathbf{J}(x, y, z)$ the excitation current density at the given frequency, $Z_{0}$ the vacuum impedance, $k_{0}=\omega / c_{0}, \mu_{\mathrm{r}}$ the relative permeability, and $\varepsilon_{\mathrm{r}}$ the relative permittivity.

The surface integral term in (6) is treated according to the respective boundary condition. On a perfectly conducting wall, the trivial condition $\mathbf{n} \times \nabla \times\left.\underline{\mathbf{E}}\right|_{\partial \boldsymbol{\Omega}_{\mathrm{pec}}}=\mathbf{0}$ is imposed. Resistive cavity walls are modeled by means of a Leontovich type surface impedance condition as

$$
\mathbf{n} \times \nabla \times\left.\underline{\mathbf{E}}\right|_{\partial \boldsymbol{\Omega}_{\text {sibc }}}=-\frac{j \omega \mu_{0}}{\underline{Z}_{\mathrm{s}}(\omega)} \mathbf{n} \times \mathbf{n} \times\left.\underline{\mathbf{E}}\right|_{\partial \mathbf{\Omega}_{\text {sibc }}},
$$

where $\mu_{0}$ is the permeability of free space and $\underline{Z}_{\mathrm{S}}(\omega)$ the surface impedance of the wall [26].

On a waveguide port boundary $\partial \boldsymbol{\Omega}_{\text {port }}$, the electromagnetic field solution is required as a superposition of incident and reflected fields [25]:

$$
\begin{aligned}
\mathbf{n} \times \nabla \times\left.\underline{\mathbf{E}}\right|_{\partial \boldsymbol{\Omega}_{\text {port }}}= & \mathbf{n} \times \nabla \times\left.\underline{\mathbf{E}}^{\mathrm{inc}}\right|_{\partial \boldsymbol{\Omega}_{\mathrm{port}}} \\
& +\sum_{m=1}^{M^{\mathrm{TE}}} \underline{r}_{m}^{\mathrm{TE}} \underline{\gamma}_{m}^{\mathrm{TE}} \mathbf{e}_{m}^{\mathrm{TE}}+\sum_{m=1}^{M^{\mathrm{TM}}} \underline{r}_{m}^{\mathrm{TM}} \frac{-k_{0}^{2}}{\underline{\gamma}_{m}^{\mathrm{TM}}} \mathbf{e}_{m}^{\mathrm{TM}},
\end{aligned}
$$

where $\mathbf{e}_{m}^{\mathrm{TE}}=\mathbf{e}_{m}^{\mathrm{TE}}(x, y)$ and $\mathbf{e}_{m}^{\mathrm{TM}}=\mathbf{e}_{m}^{\mathrm{TM}}(x, y)$ are the modal functions at $\partial \boldsymbol{\Omega}_{\text {port }}$ corresponding to the TE- and TM-modes with the propagation constants $\underline{\gamma}_{m}^{\mathrm{TE}}$ and $\underline{\gamma}_{m}^{\mathrm{TM}}$, respectively. Furthermore, $\underline{r}_{m}^{\mathrm{TE}}$ and $\underline{r}_{m}^{\mathrm{TM}}$ are the corresponding reflection coefficients for each waveguide mode at $\partial \boldsymbol{\Omega}_{\text {port }}$.

Of interest for the present discussion is the incident field $\underline{\mathbf{E}}^{\text {inc }}=\underline{\mathbf{E}}^{\text {inc }}\left(x, y, z_{\text {port }}\right)$ on a waveguide boundary corresponding to the in- and outgoing beam pipes, located at $z_{\min }$ and $z_{\max }$, respectively. In the special case of an ultrarelativistic beam moving along the $z$-axis with the speed of light in vacuum $(\beta \rightarrow 1)$, the incoming field is obtained by the solution of a $2 \mathrm{D}$ electrostatic problem

$$
\begin{gathered}
\underline{\mathbf{E}}^{\mathrm{inc}}(x, y)=-\nabla_{\perp} \varphi(x, y), \\
-\nabla_{\perp}^{2} \varphi(x, y)=\frac{\varrho(x, y)}{\varepsilon_{0}},
\end{gathered}
$$

on $\partial \boldsymbol{\Omega}_{\text {port }}$, where $\varrho(x, y)=\underline{J}_{z}\left(x, y, z_{\text {port }}\right) / c_{0}$ is the charge density on the waveguide boundary plane. For a given beam current, the solution of (10) can be performed in a preprocessing step and then incorporated in the boundary condition (8).

Given the formulation (2)-(5) and (6)-(10), the coefficients of the generalized matrix can be obtained by solving a full electromagnetic field problem for every row of (1). Each of these solutions corresponds to either the beam current excitation or to a modal field excitation on each of the waveguide ports of the structure. This procedure needs to be repeated for all frequency points considered. Due to this complexity, the calculation of generalized beam coupling matrices for the individual substructures of the system represents the computationally most intensive part of the method.

\section{The CSC $^{\text {BEAM }}$ approach \\ 1. Decomposition of the structure}

The decomposition of the chain is conducted in $\mathrm{CSC}^{\mathrm{BEAM}}$ so that the decomposition planes are normal with respect to the propagation direction of the beam.

Figure 1 depicts the decomposition of a beamline section as an example. The beam is indicated by the blue ellipses and the blue line. The planes used for the decomposition of the structure into segments are depicted as dotted red lines. In addition to the external waveguide ports of the full structure (depicted by the red solid lines), waveguide ports are assigned to the interfaces between neighboring segments (located at the red dotted lines). These ports are referred to as internal waveguide ports. At each port a finite number of waveguide modes is considered. As shown in Fig. 2, each considered mode at a port corresponds to one terminal with an incident wave amplitude $\underline{a}_{r, p, m}$ and a scattered wave amplitude $\underline{b}_{r, p, m}$. On both sides of the interface, the same number of waveguide modes is taken into account.

The number of waveguide modes considered on internal ports is a critical issue. On the one hand, the number of these modes should be small so that the number of excitations to be computed in (1) is small and the size of generalized beam coupling matrices for each of the segments remains manageable. On the other hand, the number of internal waveguide modes must be large enough 


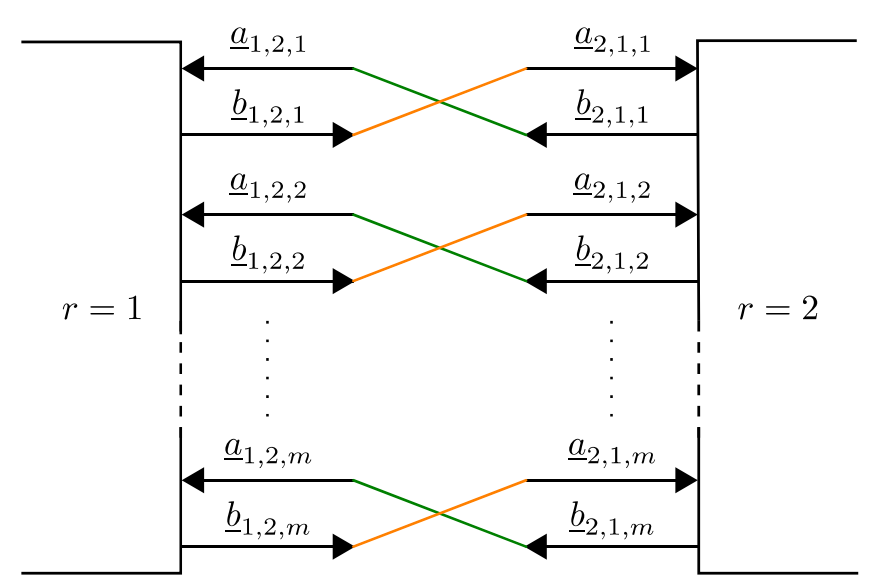

FIG. 2. Representation of the cutplane between Segment 1 and Segment 2 in Fig. 1 as an example. The segments are depicted in terms of white boxes. By way of illustration $m$ waveguide modes are considered at the cutplane. These modes are represented as terminals of both white boxes. Each terminal is equipped with the amplitude of incident waves $\underline{a}_{r, p, m}$ and the amplitude of scattered waves $\underline{b}_{r, p, m}$. The subscript $r, p, m$ denotes that the quantity belongs to the $m$ th mode at the $p$ th port of the $r$ th segment. The green and orange lines indicate the concatenation.

to accurately describe the total electromagnetic field at the interface between neighboring segments. There is no closed formula for the required number of modes as this strongly depends on geometry. As rule of thumb all waveguide modes with cutoff frequencies smaller than the maximum considered frequency $f_{\max }$ are required. In addition, a finite number of evanescent waveguide modes must be considered as well. Generally, the number of these evanescent modes depends on their attenuation constant and on the geometry of the transition region between the two segments. For a transition region resembling a long pipe of constant cross section, evanescent modes with large cutoff frequencies and therefore large attenuation constants will decay quickly and need not be considered. The shorter the transition region, the more evanescent modes need to be considered. Generally, the number of considered modes on internal ports is a parameter of the method that needs to be determined by numerical convergence tests.

\section{Concatenation of the elements}

In a first step, the dynamical properties of all segments are collated as

$$
\underline{\tilde{\mathbf{s}}}_{\mathrm{blk}} \underline{\tilde{\mathbf{a}}}_{\mathrm{blk}}=\underline{\tilde{\mathbf{b}}}_{\mathrm{blk}},
$$

where

$$
\underline{\tilde{\mathbf{S}}}_{\mathrm{blk}}=\operatorname{diag}\left(\underline{\tilde{\mathbf{S}}}_{1}, \underline{\mathbf{S}}_{2}, \ldots, \tilde{\tilde{\mathbf{S}}}_{R}\right)
$$

is a block diagonal matrix holding the generalized matrices (1) of each individual segment. The input vector

$$
\underline{\tilde{\mathbf{a}}}_{\mathrm{blk}}=\left(\begin{array}{c}
\underline{\tilde{\mathbf{a}}}_{1} \\
\underline{\tilde{\mathbf{a}}}_{2} \\
\vdots \\
\underline{\tilde{\mathbf{a}}}_{R}
\end{array}\right)
$$

contains all the generalized input vectors of each segment, and

$$
\underline{\tilde{\mathbf{b}}}_{\mathrm{blk}}=\left(\begin{array}{c}
\tilde{\tilde{\mathbf{b}}}_{1} \\
\underline{\tilde{\mathbf{b}}}_{2} \\
\vdots \\
\tilde{\tilde{\mathbf{b}}}_{R}
\end{array}\right)
$$

is a vector containing all the generalized output vectors of each segment as defined in (1).

Next, the ordering of $\underline{\mathbf{a}}_{b l k}$ and $\underline{\tilde{\mathbf{b}}}_{b l k}$ is modified by means of a permutation matrix $\mathbf{P}$, so that

$$
\begin{gathered}
\mathbf{P} \underline{\mathbf{a}}_{\mathrm{blk}}=\underline{\tilde{\mathbf{a}}}_{\mathrm{stt}}=\left(\begin{array}{c}
\underline{\mathbf{a}}_{\mathrm{int}} \\
\underline{\mathbf{a}}_{\mathrm{ext}} \\
\underline{\mathbf{i}}
\end{array}\right), \\
\mathbf{P} \underline{\tilde{\mathbf{b}}}_{\mathrm{blk}}=\underline{\tilde{\mathbf{b}}}_{\mathrm{srt}}=\left(\begin{array}{c}
\underline{\mathbf{b}}_{\mathrm{int}} \\
\underline{\mathbf{b}}_{\mathrm{ext}} \\
\underline{\mathbf{v}}
\end{array}\right)
\end{gathered}
$$

is obtained. The size of the permutation matrix $\mathbf{P}$ is determined by the number of ports and the number of waveguide modes at the respective ports of all segments. The complex-valued vectors $\underline{\mathbf{a}}_{\text {int }} \in \mathbb{C}^{N_{\text {int }} \times 1}$ and $\underline{\mathbf{b}}_{\text {int }} \in$ $\mathbb{C}^{N_{\text {int }} \times 1}$ in (15) and (16) comprise, respectively, incident and scattered wave amplitudes of the internal ports. The vectors $\underline{\mathbf{a}}_{\mathrm{ext}} \in \mathbb{C}^{N_{\mathrm{ext}} \times 1}$ and $\underline{\mathbf{b}}_{\mathrm{ext}} \in \mathbb{C}^{N_{\mathrm{ext}} \times 1}$ in (15) and (16) contain, respectively, incident and scattered wave amplitudes of external ports. Here, $N_{\text {int }}$ and $N_{\text {ext }}$ denote the total number of internal and external waveguide modes, respectively. The vectors $\underline{\mathbf{i}}$ and $\underline{\mathbf{v}}$ collate the beam currents and the beam voltages in each of the $R$ segments:

$$
\begin{aligned}
\underline{\mathbf{i}} & =\left(\begin{array}{llll}
\underline{i}_{1} & \underline{i}_{2} & \ldots & \underline{i}_{R}
\end{array}\right)^{\mathrm{T}}, \\
\underline{\mathbf{v}} & =\left(\begin{array}{llll}
\underline{v}_{1} & \underline{v}_{2} & \cdots & \underline{v}_{R}
\end{array}\right)^{\mathrm{T}} .
\end{aligned}
$$

Replacing the vectors $\underline{\tilde{\mathbf{a}}}_{b l k}$ and $\underline{\tilde{\mathbf{b}}}_{\text {blk }}$ in (11) by their sorted counterparts defined in (15) and (16) and using the orthogonality of permutation matrices yields

$$
\underbrace{\mathbf{P} \underline{\tilde{\mathbf{S}}}_{\mathrm{blk}} \mathbf{P}^{\mathrm{T}}}_{\underline{\mathbf{G}}} \tilde{\tilde{\mathbf{a}}}_{\mathrm{srt}}=\underline{\tilde{\mathbf{b}}}_{\mathrm{str}} .
$$


Next, $\underline{\mathbf{G}}$ is written as a block matrix, so that (19) becomes

$$
\underbrace{\left(\begin{array}{ll}
\underline{\mathbf{G}}_{11} & \underline{\mathbf{G}}_{12} \\
\underline{\mathbf{G}}_{21} & \underline{\mathbf{G}}_{22}
\end{array}\right)}_{\underline{\mathbf{G}}} \underbrace{\left(\begin{array}{c}
\underline{\mathbf{a}}_{\mathrm{int}} \\
\underline{\mathbf{a}}_{\mathrm{ext}} \\
\underline{\mathbf{i}}
\end{array}\right)}_{\underline{\tilde{\mathbf{a}}}_{\mathrm{st}}}=\underbrace{\left(\begin{array}{c}
\underline{\mathbf{b}}_{\mathrm{int}} \\
\underline{\mathbf{b}}_{\mathrm{ext}} \\
\underline{\mathbf{v}}
\end{array}\right)}_{\underline{\tilde{\mathbf{b}}}_{\mathrm{st}}},
$$

where

$$
\begin{array}{lll}
\underline{\mathbf{G}}_{11} \in \mathbb{C}^{N_{\text {int }} \times N_{\text {int }},} & \underline{\mathbf{G}}_{12} \in \mathbb{C}^{N_{\text {int }} \times\left(N_{\mathrm{ext}}+R\right)}, \\
\underline{\mathbf{G}}_{21} \in \mathbb{C}^{\left(N_{\mathrm{ext}}+R\right) \times N_{\text {int }},} & \underline{\mathbf{G}}_{22} \in \mathbb{C}^{\left(N_{\mathrm{ext}}+R\right) \times\left(N_{\mathrm{ext}}+R\right)} .
\end{array}
$$

This enables the expression of (20) by means of

$$
\begin{aligned}
& \underline{\mathbf{G}}_{11} \underline{\mathbf{a}}_{\mathrm{int}}+\underline{\mathbf{G}}_{12}\left(\begin{array}{c}
\underline{\mathbf{a}}_{\mathrm{ext}} \\
\underline{\mathbf{i}}
\end{array}\right)=\underline{\mathbf{b}}_{\mathrm{int}}, \\
& \underline{\mathbf{G}}_{21} \underline{\mathbf{a}}_{\mathrm{int}}+\underline{\mathbf{G}}_{22}\left(\begin{array}{c}
\underline{\mathbf{a}}_{\mathrm{ext}}^{\mathbf{i}} \\
\underline{\mathbf{i}}
\end{array}\right)=\left(\begin{array}{c}
\underline{\mathbf{b}}_{\mathrm{ext}} \\
\underline{\mathbf{v}}
\end{array}\right) .
\end{aligned}
$$

The coupling of the internal quantities is enforced by

$$
\mathbf{F} \underline{\mathbf{a}}_{\text {int }}=\underline{\mathbf{b}}_{\text {int }} .
$$

Similarly to the matrix $\mathbf{P}$, the feedback matrix $\mathbf{F} \in \mathbb{N}^{N_{\text {int }} \times N_{\text {int }}}$ is a permutation matrix that couples the incoming waves in each segment with the corresponding outgoing waves of the previous one. This matrix is determined by the permutation matrix $\mathbf{P}$, the topology of the segments to be coupled, and by the number of internal terminals.

Using (23) to replace $\underline{\mathbf{b}}_{\text {int }}$ in (21) and sorting for the internal incident wave amplitudes yields

$$
\underline{\mathbf{a}}_{\mathrm{int}}=\left(\mathbf{F}-\underline{\mathbf{G}}_{11}\right)^{-1} \underline{\mathbf{G}}_{12}\left(\begin{array}{c}
\underline{\mathbf{a}}_{\mathrm{ext}} \\
\underline{\mathbf{i}}
\end{array}\right) \text {. }
$$

Employing this statement to substitute the internal incident waves $\underline{\mathbf{a}}_{\text {int }}$ in (22) gives

$$
\left[\underline{\mathbf{G}}_{22}+\underline{\mathbf{G}}_{21}\left(\mathbf{F}-\underline{\mathbf{G}}_{11}\right)^{-1} \underline{\mathbf{G}}_{12}\right]\left(\begin{array}{c}
\underline{\mathbf{a}}_{\mathrm{ext}} \\
\underline{\mathbf{i}}
\end{array}\right)=\left(\begin{array}{c}
\underline{\mathbf{b}}_{\mathrm{ext}} \\
\underline{\mathbf{v}}
\end{array}\right) .
$$

On account of charge conservation and the rigid beam assumption, the magnitude of the beam current in every segment is the same. However, while moving in the longitudinal direction along the chain of segments, phase of the beam current in the respective segments is shifted as

$$
\underline{i}_{r}=\underline{i} \exp (-j \underbrace{\frac{\omega z_{r}}{\beta c_{0}}}_{\varphi_{r}}),
$$

with the position of the entrance of the $r$ th segment

$$
z_{r}=\sum_{k=1}^{r-1} L_{r}
$$

(refer to Fig. 1) and $L_{r}$ its length. Expressing (26) for all $R$ currents gives

$$
\underbrace{\left(\begin{array}{c}
\underline{i}_{1} \\
\underline{i}_{2} \\
\vdots \\
\underline{i}_{R}
\end{array}\right)}_{\underline{\mathbf{i}}}=\underbrace{\left(\begin{array}{c}
1 \\
\exp \left(-j \varphi_{2}\right) \\
\vdots \\
\exp \left(-j \varphi_{R}\right)
\end{array}\right)}_{\underline{\mathbf{d}}} \underline{i}
$$

with the complex-valued vector $\underline{\mathbf{d}} \in \mathbb{C}^{R \times 1}$. The different phases of the beam currents in every segment are now accounted for in (25) by

$$
\left(\begin{array}{c}
\underline{\mathbf{a}}_{\mathrm{ext}} \\
\underline{\mathbf{i}}
\end{array}\right)=\underline{\mathbf{M}}\left(\begin{array}{c}
\underline{\mathbf{a}}_{\mathrm{ext}} \\
\underline{i}
\end{array}\right)
$$

with the block diagonal matrix

$$
\underline{\mathbf{M}}=\operatorname{diag}(\mathbf{I}, \underline{\mathbf{d}}) \in \mathbb{C}^{\left(N_{\mathrm{ext}}+R\right) \times\left(N_{\mathrm{ext}}+1\right)} .
$$

Here, I denotes the identity matrix.

The total voltage $\underline{v}$ experienced by the beam traversing the complete chain of segments can be written by splitting (5) into individual integrals for each segment:

$$
\underline{v}=\sum_{r=1}^{R} \int_{z_{r}}^{z_{r}+L_{r}} \underline{E}_{z}\left(x_{\mathrm{b}}, y_{\mathrm{b}}, z\right) \exp \left(\frac{j \omega z}{\beta c_{0}}\right) \mathrm{d} z .
$$

Substituting $\hat{z}=z-z_{r}$ gives

$$
\underline{v}=\sum_{r=1}^{R} \underbrace{\int_{0}^{L_{r}} \underline{E}_{r, z}\left(x_{\mathrm{b}}, y_{\mathrm{b}}, \hat{z}\right) \exp \left(\frac{j \omega \hat{z}}{\beta c_{0}}\right) \mathrm{d} \hat{z}}_{\underline{v}_{r}} \times \exp \left(\frac{j \omega z_{r}}{\beta c_{0}}\right),
$$

where $\underline{v}_{r}$ is the voltage induced on the beam in segment $r$ by the current $\underline{i}$ without taking into account the phase shift in the voltage resulting from the shift of the segment. Thus, $\underline{v}_{r}$ is the usual beam voltage corresponding to the current $\underline{i}$ as appears from the generalized coupling matrix formulation (1) for the respective segment. Relation (32) can be further written as 


$$
\underline{\mathbf{d}}^{\mathrm{H}} \underbrace{\left(\begin{array}{c}
\underline{v}_{1} \\
\underline{v}_{2} \\
\vdots \\
\underline{v}_{R}
\end{array}\right)}_{\underline{\mathbf{v}}}=\underline{v},
$$

whereas $\underline{\mathbf{d}}^{\mathrm{H}}$ is the conjugate transposed of $\underline{\mathbf{d}}$ as defined in (28).

As a result of (33), the right-hand side of (25) can be replaced by

$$
\underline{\mathbf{M}}^{\mathrm{H}}\left(\begin{array}{c}
\underline{\mathbf{b}}_{\mathrm{ext}} \\
\underline{\mathbf{v}}
\end{array}\right)=\left(\begin{array}{c}
\underline{\mathbf{b}}_{\mathrm{ext}} \\
\underline{v}
\end{array}\right) \text {. }
$$

Multiplying (25) from the left-hand side with $\underline{\mathbf{M}}^{\mathrm{H}}$ and replacing the excitation vector as well as the right-hand side by (29) and (34), results in

$$
\underline{\tilde{\mathbf{s}}}_{\mathrm{csc}} \underbrace{\left(\begin{array}{c}
\underline{\mathbf{a}}_{\mathrm{ext}} \\
\underline{i}
\end{array}\right)}_{\underline{\tilde{\mathbf{a}}}_{\mathrm{ext}}}=\underbrace{\left(\begin{array}{c}
\underline{\mathbf{b}}_{\mathrm{ext}} \\
\underline{v}
\end{array}\right)}_{\underline{\tilde{\mathbf{b}}}_{\mathrm{ext}}},
$$

with the generalized beam coupling matrix

$$
\underline{\tilde{\mathbf{S}}}_{\mathrm{csc}}=\underline{\mathbf{M}}^{\mathrm{H}}\left[\underline{\mathbf{G}}_{22}+\underline{\mathbf{G}}_{21}\left(\mathbf{F}-\underline{\mathbf{G}}_{11}\right)^{-1} \underline{\mathbf{G}}_{12}\right] \underline{\mathbf{M}}
$$

of the concatenated structure. Following definition (1), the coefficient in the last row of the last column is the beam impedance of the entire structure.

\section{Reconstruction of field distributions}

Subsequently, the field reconstruction is discussed for electric fields. The approach for magnetic fields is analogous. For the reconstruction of the field distributions in concatenated structures, the fields in the segments have to be computed and stored for all possible excitations at the waveguide ports and the beam excitation. Note that these computations need to be performed anyhow to determine the generalized beam coupling matrix (1) of each segment. The electric field distribution in the segment $r$ is given by

$$
\underline{\mathbf{E}}_{r}(x, y, z)=\sum_{k=1}^{N_{r}+1} \underline{\mathbf{E}}_{r, k}(x, y, z) \underline{\tilde{a}}_{r, k},
$$

where $\underline{\tilde{a}}_{r, k}$ is the $k$ th coefficient of the generalized excitation vector $\underline{\tilde{\mathbf{a}}}_{r}$ of the $r$ th segment, $\underline{\mathbf{E}}_{r, k}(x, y, z)$ the electric field strength in the $r$ th segment resulting from the excitation $\underline{\tilde{a}}_{r, k}=1$ while the remaining coefficients in $\underline{\tilde{a}}_{r}$ equal zero, and $N_{r}$ the number of terminals corresponding to all waveguide modes at the various ports of the $r$ th segment. The total field distribution in the concatenated chain is therefore described by

$$
\underline{\mathbf{E}}(x, y, z)=\sum_{r=1}^{R} \sum_{k=1}^{N_{r}+1} \underline{\mathbf{E}}_{r, k}(x, y, z) \underline{\tilde{a}}_{r, k} .
$$

In order to finally evaluate (38), all coefficients $\underline{\tilde{a}}_{r, k}$ which are collected in $\underline{\mathbf{a}}_{b l k}$ need to be determined in terms of the external excitation (that can be the beam or a waveguide mode at an external port). The relationship between these quantities is given by

$$
\underline{\tilde{\mathbf{a}}}_{\mathrm{blk}}=\mathbf{P}^{\mathrm{T}} \underline{\tilde{\mathbf{a}}}_{\mathrm{stt}}=\mathbf{P}^{\mathrm{T}}\left(\begin{array}{c}
\underline{\mathbf{a}}_{\mathrm{int}} \\
\underline{\mathbf{a}}_{\mathrm{ext}} \\
\underline{\mathbf{i}}
\end{array}\right)=\mathbf{P}^{\mathrm{T}} \underline{\mathbf{D}}\left(\begin{array}{c}
\underline{\mathbf{a}}_{\mathrm{ext}} \\
\underline{i}
\end{array}\right),
$$

with the block matrix

$$
\underline{\mathbf{D}}=\left(\begin{array}{c}
\left(\mathbf{F}-\underline{\mathbf{G}}_{11}\right)^{-1} \underline{\mathbf{G}}_{12} \underline{\mathbf{M}} \\
\underline{\mathbf{M}}
\end{array}\right),
$$

which results from exploiting (15), (24), and (29).

\section{NUMERICAL COMPUTATIONS}

\section{A. Implementation of the scheme}

The scheme is implemented in two steps. First, a FEMbased frequency domain impedance solver is developed that enables the computation of the generalized beam coupling matrices. Second, the concatenation scheme described above is applied using these matrices.

So far, the solution of the full 3D Maxwell problem in the frequency domain in the presence of a beam passing through in- and outgoing pipes has been presented in the literature only for frequencies below cutoff [27], for rotational symmetric structures [28] or for planar geometries in 2D [29]. Therefore, the development of a dedicated field solver based on the formulation (2)-(5) and (6)-(10) was necessary. For the finite element discretization, the highorder hierarchic basis functions proposed in [24] were adopted and further implemented in an in-house simulation platform [30].

One of the main difficulties in the simulation of electromagnetic fields in the presence of particle beams stems from the singularity of the transverse electric field components on the beam axis. Due to this singularity, the numerical solution introduces huge errors in the longitudinal field and thus in the beam impedance. This effect was early recognized in [31], where it was shown that a disparity by orders of magnitude between the transverse and longitudinal field components leads to large numerical noise in wakefield simulations with unstructured grids. Furthermore, it was shown that using a regular Cartesian mesh allows to fully decouple transverse and longitudinal 

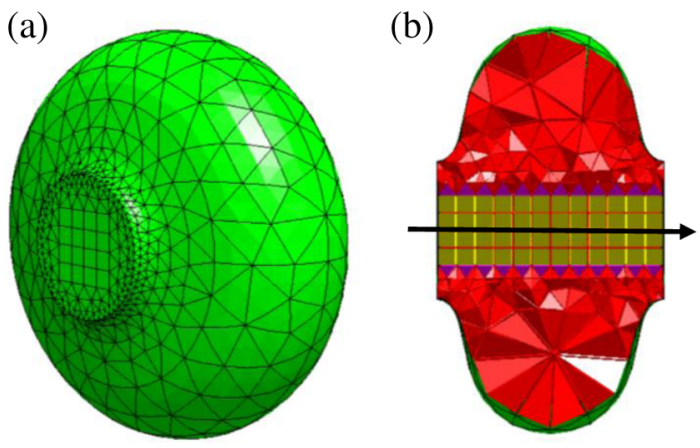

FIG. 3. (a) Outer view of the hybrid mesh used to discretize a single TESLA cell. (b) Cut view of the mesh featuring the Cartesian elements in the beam region, the curved tetrahedral elements resolving the cavity wall and the connecting pyramidal layer in between.

error contributions, resulting in noisefree simulations. In this work, a similar discretization idea is used. A hybrid mesh discretization is applied consisting of a uniform Cartesian submesh around the beam and of an unstructured submesh of curved tetrahedral elements resolving the geometry of the cavity. Mesh conformity is ensured by applying a layer of pyramidal elements at the interface between the two submeshes. Such mesh constructions can be generated in an automated fashion using the meshing platform SALOME [32]. The hybrid mesh approach is applied for all considered examples in the paper. Figure 3 depicts an example of such a hybrid mesh that is applied for the discretization of single cell of the $1.3 \mathrm{GHz}$ TESLA cavity (cf. Sec. III C).

The concatenation procedure is implemented as a script in the Matlab R2017 platform [33]. The script reads in the generalized beam coupling matrices of all segments sampled at the frequencies of interest in addition to topology information. The latter specifies the ports to be concatenated and the number of waveguide modes considered for each port. Based on this information, the matrices $\mathbf{P}$ and $\mathbf{F}$ are constructed. In addition, the lengths of the individual segments are required to determine the matrix $\underline{\mathbf{M}}$. The scripts and the raw data for the following application examples are provided as Supplemental Material in [34].

\section{B. Cylindrical cavity}

The simple cylindrical cavity consisting of two coupled perfectly conducting segments shown in Fig. 4 is used as a fundamental proof of principle for the proposed approach. This example allows for estimating the ability of CSC $^{\text {BEAM }}$ to reproduce resonances of the concatenated structure whereas its segments are non-resonant (above the cutoff frequencies of the corresponding waveguide modes). The particle beam exciting the structure is defined on the symmetry axis. The in- and outgoing beam pipes are represented by the two (external) waveguide ports marked

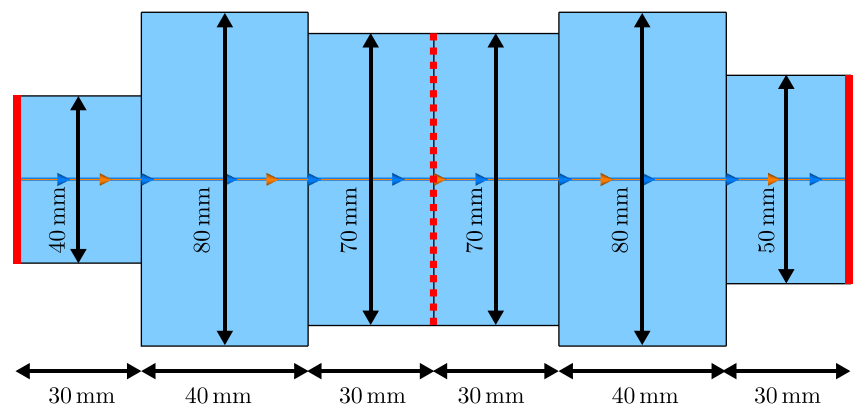

FIG. 4. Vacuum model of a rotational symmetric coupled cavity constructed by six cylinders. The structure is visualized with CST [7] using a cutplane. The decomposition of the structure takes place at the red dotted line. The beam of charged particles is depicted by orange/blue lines and orange/blue cones and traverses the structure on the symmetry axis. The external waveguide ports are illustrated by red solid lines at the beam pipes of the structure.

as red solid lines at both ends of the structure. The cutplane used to decompose the structure into two segments is indicated in Fig. 4 as red dotted line.

For generality, the radii of the in- and outgoing beam pipes are chosen to be different. The radius at the intermediate beam pipe connecting the two segments is chosen to be much larger than the beam pipe radii, so that higher-order waveguide modes may contribute to the coupling between the segments. Moreover, the two segments are nonresonant above the cutoff frequency of the lowest TM mode in the in- and outgoing beam pipes whereas resonant modes with large quality factors exist in the complete structure.

The generalized beam coupling matrices (1) are computed for each segment on 161 equidistant frequency samples in the interval $2 \mathrm{GHz}$ to $10 \mathrm{GHz}$. At the external ports solely the $\mathrm{TM}_{01}$ waveguide modes are considered. Their cutoff frequencies are $5.74 \mathrm{GHz}$ and $4.59 \mathrm{GHz}$ for the in- and outgoing pipes, respectively. At the decomposition plane two waveguide modes are considered, namely the $\mathrm{TM}_{01}$ and the $\mathrm{TM}_{02}$. Their cutoff frequencies are $3.28 \mathrm{GHz}$ and $7.52 \mathrm{GHz}$, respectively. Therefore, the generalized matrix for the complete structure has three rows and columns whereas the generalized matrices for the individual segments have four rows and four columns each. Note that for the given example $N_{\text {ext }}=2$ and $N_{\text {int }}=4$.

Figure 5 presents the computed beam impedances. The red solid lines arise from the direct impedance computation of the complete structure and are used as reference curves. The solid blue curves result from CSC $^{\mathrm{BEAM}}$ with no internal waveguide modes considered at the decomposition plane. Thus, it corresponds to a simple addition of the beam impedances of the two cavities. The orange curves arise from $\mathrm{CSC}^{\mathrm{BEAM}}$ when a single internal waveguide mode $\left(\mathrm{TM}_{01}\right)$ is considered whereas the purple dashed curves result from $\mathrm{CSC}^{\mathrm{BEAM}}$ with two waveguide modes $\left(\mathrm{TM}_{01}\right.$ and $\mathrm{TM}_{02}$ ) considered at the decomposition plane. 


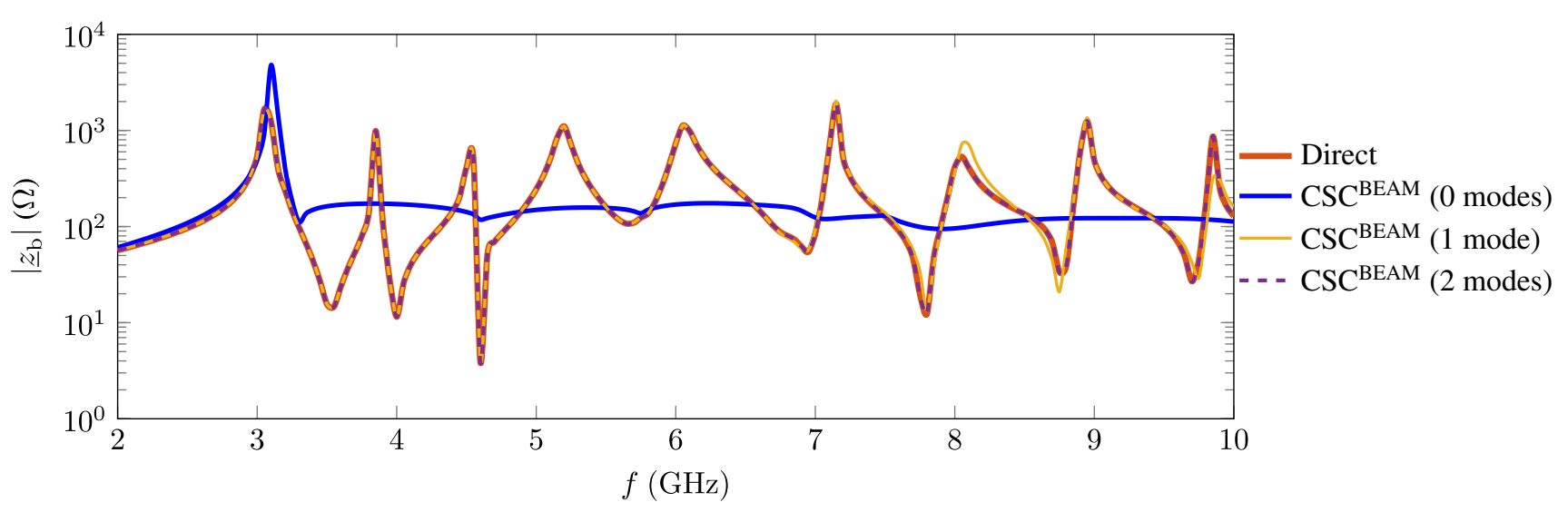

(a)

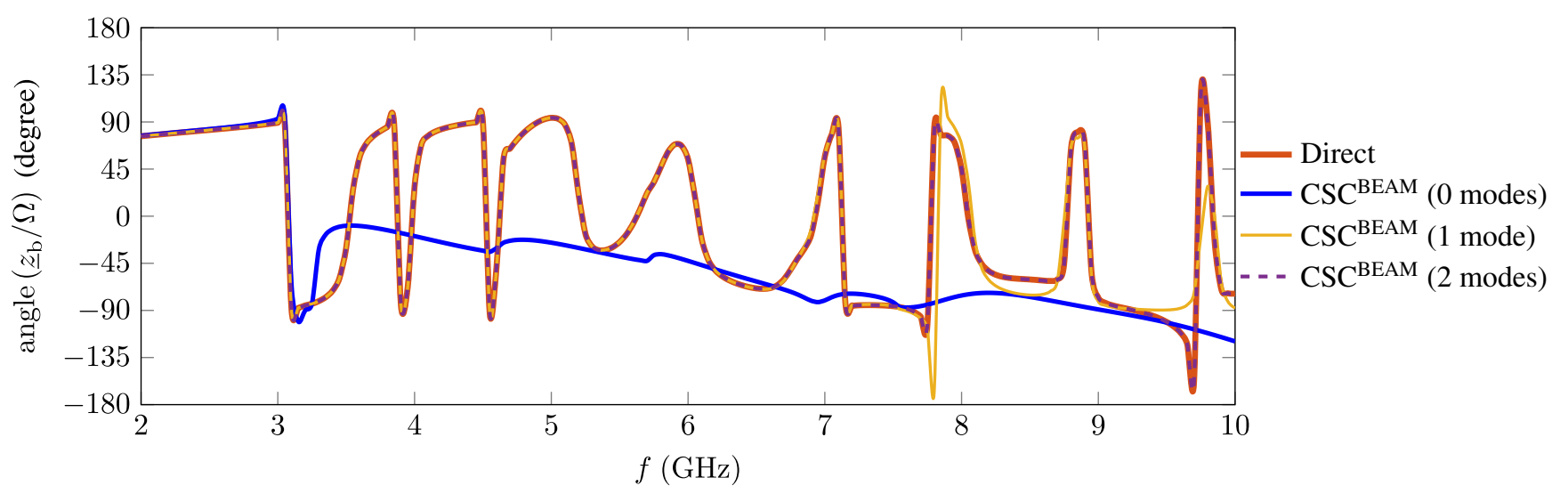

(b)

FIG. 5. Beam impedance of the rotational symmetric structure depicted in Fig. 4: (a) absolute values and (b) phases of the beam impedance. The red solid lines depict the impedance resulting from a direct computation, the blue solid lines from a simple phaseaccounting addition of the impedances of both segments (i.e., zero waveguide modes considered at the decomposition plane), the orange solid lines from the proposed approach with solely the $\mathrm{TM}_{01}$ mode considered at the decomposition plane, and the purple dashed lines from the proposed approach with the $\mathrm{TM}_{01}$ and the $\mathrm{TM}_{02}$ mode considered at the decomposition plane.

As seen in the figure, the approximation of the total impedance by simply adding the individual impedances of the segments completely fails above the cutoff frequency $3.28 \mathrm{GHz}$ of the $\mathrm{TM}_{01}$ mode at the decomposition plane as expected. Including a single coupling mode $\left(\mathrm{TM}_{01}\right)$ in the formulation already shows a much better agreement with the reference curve over the entire frequency interval. However, small deviations are observed beyond the cutoff frequency $7.52 \mathrm{GHz}$ of the $\mathrm{TM}_{02}$ mode of the connecting pipe. Including this mode in the $\operatorname{CSC}^{\mathrm{BEAM}}$ computation results in a perfect agreement between the beam impedance delivered by the direct run and the concatenation. Note that the comparably small number of waveguide modes required at the decomposition plane for the concatenation results from the symmetry of this academic example and its symmetric excitation. Generally, many more waveguide modes are required for a reasonable field expansion as will be shown in Sec. III C.

Figure 6 depicts the normalized longitudinal component of the real part of the electric field strength in the circular cavity under study. All fields result from an excitation at $3.85 \mathrm{GHz}$ by solely the beam current, i.e., the waveguide ports at the ends of the beam pipes are not excited. This frequency is chosen on purpose. The full structure is resonant at this frequency (cf. Fig. 5) whereas the individual segments are not. Figure 6(a) is obtained by a computation of the full structure and serves as reference. Figure 6(b) shows the field distribution arising from the beam excitation in each segment separately. Figure 6(c) results from reconstructing the fields with $\mathrm{CSC}^{\mathrm{BEAM}}$ as it is discussed in Sec. II C 3. Figures 6(a) and 6(c) show a very good agreement and a resonant mode in both segments is clearly visible. In contrast, this resonant mode is not observable in Fig. 6(b).

\section{TESLA cavity with couplers}

The impedance calculation of the TESLA cavity with higher-order mode and input couplers in Fig. 7 is employed as a more advanced application example for the proposed 


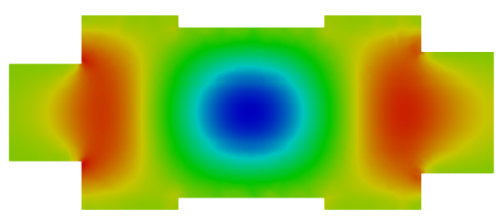

(a)

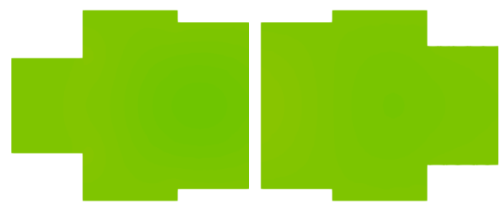

(b)

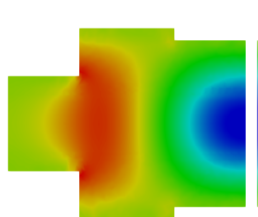

(c)

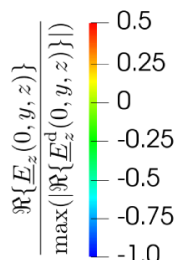

(d)

FIG. 6. Real part of the longitudinal electric field strength in the rotational symmetric structure shown in Fig. 4: (a) field strength arising from the direct computation (reference solution), (b) field distribution arising from an individual beam excitation in both segments without mutual coupling via electromagnetic fields, (c) field strength delivered by CSC ${ }^{\text {BEAM }}$, and (d) legend. The decomposition plane is indicated by a white bar in (b) and (c). All field strengths result from an excitation with an on-axis charged particle beam at $3.85 \mathrm{GHz}$. All three field distributions are normalized to $\left.\max \left|\operatorname{Re}\left\{\underline{E}_{z}^{\mathrm{d}}(0, y, z)\right\}\right|\right)$, whereas $\underline{E}_{z}^{\mathrm{d}}(x, y, z)$ is the longitudinal field strength from the direct computation. The plots are created with ParaView [35].

approach. Details of the geometry can be found in [36] and references therein. The particle beam is traversing on the symmetry axis of the cells. The structure is equipped with (external) waveguide ports at both beam pipe ends, i.e., at $z_{\min }$ and $z_{\max }$. The external waveguide ports are indicated by the solid red lines. The cutplanes used to decompose the complete structure into segments are indicated in Fig. 7 as red dotted lines. The decomposition results in 11 segments, namely upstream higher-order mode coupler $(r=1)$, upstream end cell $(r=2)$, mid cells $(r=3, \ldots, 9)$, downstream end cell $(r=10)$, and downstream higher-order mode coupler with input coupler $(r=11)$. Note that the input coupler is not visible in Fig. 7 due to the cutplane representation. Surface losses are not considered.

Since the midcells are identical, only five generalized beam coupling matrices need to be computed for the 11 segments constituting the full structure. Note that only the columns relevant for the computation of the beam impedance of the full structure are computed, i.e., excitations at external ports of the structure are not required. The computations are performed for 601 equidistant frequency samples in the interval $1 \mathrm{GHz}$ to $4 \mathrm{GHz}$. For the FEM discretization of each segment a hybrid mesh with approximately 9,000 hexahedral, tetrahedral, and pyramidal finite elements is used as shown in Fig. 3. At the external ports as well as at the decomposition planes 18 waveguide modes are considered: 11 modes of TE type and 7 modes of TM type. Their cutoff frequencies range from $2.25 \mathrm{GHz}$ to $7.81 \mathrm{GHz}$ for the beam pipes and from $2.51 \mathrm{GHz}$ to $8.70 \mathrm{GHz}$ for the cutplanes, so that all propagating modes as well as several evanescent modes are considered. As a result of this choice, the generalized matrices for the complete structure and for the individual segments have 37 rows and columns. In the presented example $N_{\text {ext }}=36$ external terminals and $N_{\text {int }}=360$ internal terminals exist.

Figure 8 shows the computed beam impedances. The red solid lines result from the direct impedance computation of the full structure and are therefore providing the reference curves. The blue solid lines arise from the complex-valued addition of the individual beam impedances of all segments, i.e., no coupling across the decomposition planes is modeled. The orange curves result from $\mathrm{CSC}^{\mathrm{BEAM}}$ with 18 waveguide modes considered for the field expansion at the decomposition planes.

Figure 8 depicts that the pure addition of the individual segment impedances leads to a reasonable impedance

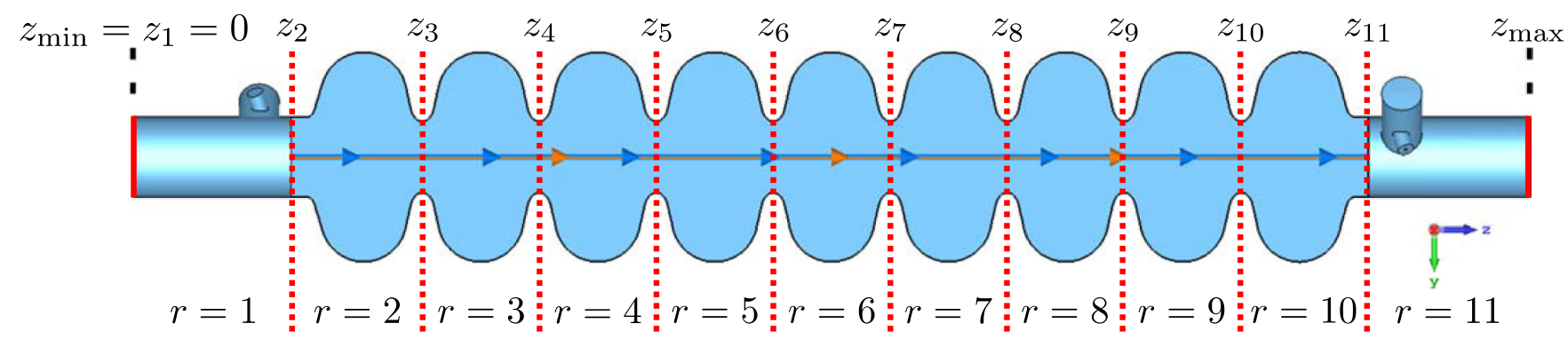

FIG. 7. Vacuum model of a TESLA cavity with higher-order mode and input couplers [36]. The charged particle beam is illustrated by orange/blue lines and orange/blue cones and is defined from $z_{\min }$ to $z_{\max }$. The $z$-coordinates at which the beam enters the $r$ th segment are denoted by $z_{r}$. Note that the beam is solely visible in the nine elliptical-shaped cells since a $y z$-cutplane is used to draw these cells. The $x y$-cutplanes employed to decompose the structure are depicted in terms of dotted red lines. The geometry is visualized using CST Studio Suite ${ }^{\circledR}$ [7]. 


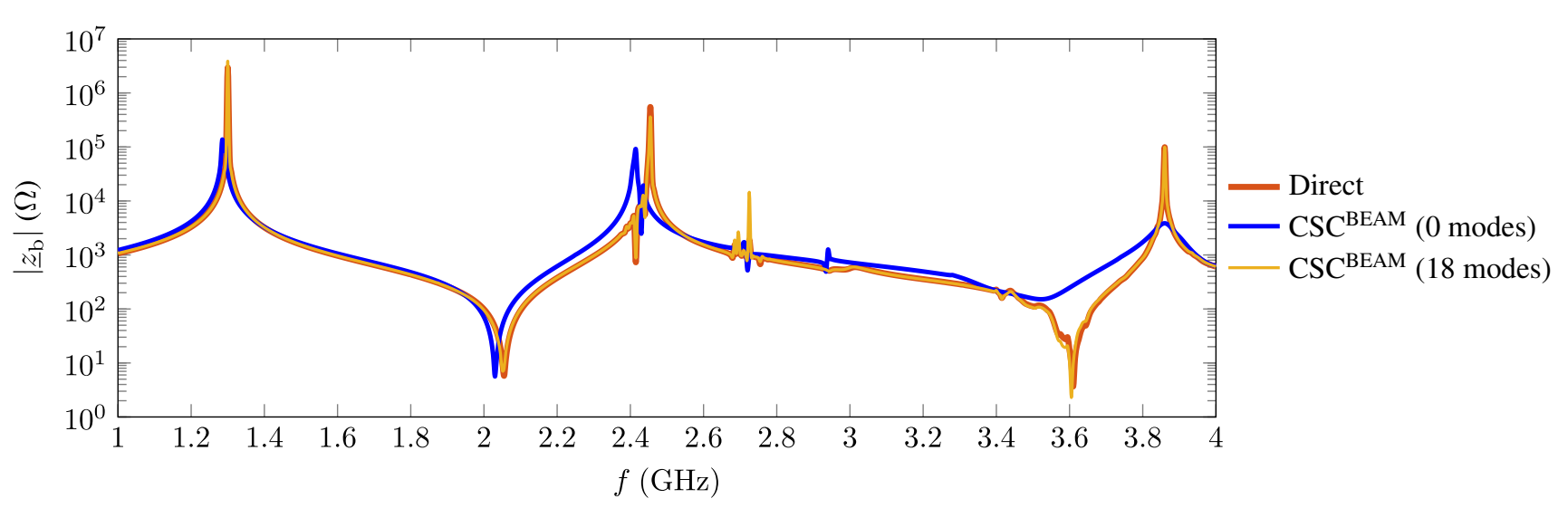

(a)

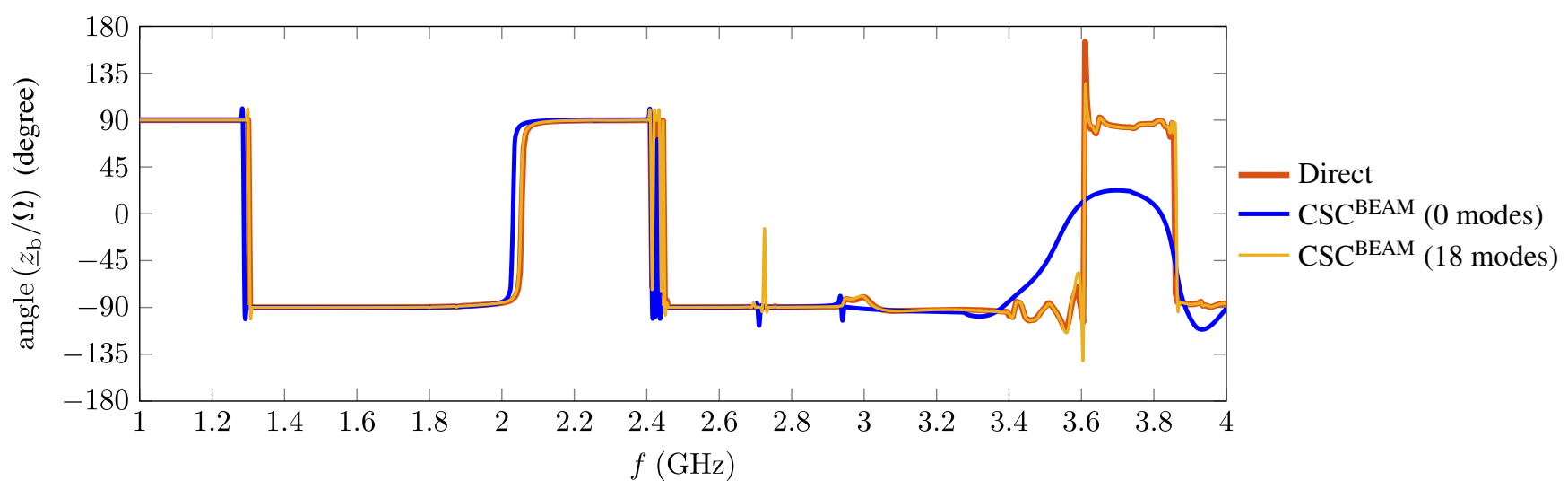

(b)

FIG. 8. Beam impedance of the TESLA cavity higher-order mode couplers and input couplers as depicted in Fig. 7: (a) absolute values and (b) phases of the beam impedance. The red solid lines depict the impedance from a direct computation, the blue solid lines by a simple phase-accounting addition of the impedances of all 11 segments (i.e., no waveguide modes considered at the decomposition planes), the orange solid lines by the proposed approach with 18 modes considered at each of the ten decomposition planes.

approximation of the full structure up to $2.2 \mathrm{GHz}$. Only small frequency shifts of the peak at $1.3 \mathrm{GHz}$ and the notch at $2.05 \mathrm{GHz}$ can be observed. Note that exclusively the $\mathrm{TM}_{01}-\pi$ mode is visible in the spectrum around $1.3 \mathrm{GHz}$. The remaining eight modes of the fundamental band are not observable on account of their small on-axis coupling impedances [37]. The approximation by a simple complex-valued impedance addition deteriorates in the interval $2.2 \mathrm{GHz}$ to $3.4 \mathrm{GHz}$, whereas it completely fails beyond $3.4 \mathrm{GHz}$. The location of the peak at $2.45 \mathrm{GHz}$ is not accurately reproduced. The small resonance features below this peak are not visible in the blue lines at all. Moreover, the blue curve shows a peak at $2.94 \mathrm{GHz}$ which is by far less distinctive in the red reference curves. The notch effect at $3.6 \mathrm{GHz}$ is not approximated at all and the quality factor of the resonance at $3.86 \mathrm{GHz}$ is significantly underestimated in the blue curves. In contrast to this, $\mathrm{CSC}^{\mathrm{BEAM}}$ delivers a extremely good approximation of the impedance over the entire considered band. Tiny features of the reference curves are observable in the orange curves as well.

\section{SUMMARY AND CONCLUSIONS}

The current article motivates the need of a method to compute beam coupling impedances of coupled accelerator parts. The article introduces a flexible scheme which allows for the computation of global impedances in electromagnetically coupled elements of particle accelerators. The new method is referred to as $\mathrm{CSC}^{\mathrm{BEAM}}$ as it is based on the generalization of CSC. The theory of the method is introduced and two validation examples are presented. The validation examples show the flexibility of the method in terms of the number of waveguide modes used for the concatenation. As far as the authors can determine, the scheme is not limited by the number of segments used for the concatenation. In fact, the computational complexity of the scheme is rather related to the number of waveguide modes employed for the coupling of the fields across the decomposition planes. Therefore, the scheme is suitable to accurately determine global impedance models of entire machines. In this way, CSC $^{\text {BEAM }}$ can help to identify missing impedance contributions in global impedance models of particle accelerators. 


\section{ACKNOWLEDGMENTS}

The research leading to these results was partly supported by the German Bundesministerium für Bildung und Forschung, Land Berlin and grants of Helmholtz Association. The authors would like to thank Ursula van Rienen from University of Rostock for carefully reading the manuscript and for making valuable proposals for its improvement.

[1] H. Wiedemann, Beam stability and vacuum chamber design, AIP Conf. Proc. 171, 10 (1988).

[2] V. G. Vaccaro, Longitudinal instability of a coasting beam above transition, due to the action of lumped discontinuities, CERN Report No. ISR-RF/66-35, 1966.

[3] V. G. Vaccaro, The birth and childhood of a couple of twin brothers, in Proceedings of ICFA Mini-Workshop on Impedances and Beam Instabilities in Particle Accelerators (Benevento, Italy, 2017), pp. 117-123.

[4] I. Zagorodnov and T. Weiland, TE/TM field solver for particle beam simulations without numerical Cherenkov radiation, Phys. Rev. Accel. Beams 8, 042001 (2005).

[5] E. Gjonaj, T. Lau, and T. Weiland, Computation of short range wake field with PBCI, in ICFA Beam Dynamics Newsletter No. 45 (2008), pp. 38-52.

[6] W. Bruns, GdfidL: A finite difference program with reduced memory and CPU usage, in Proceedings of the Particle Accelerator Conference, Vancouver, BC, Canada, 1997 (IEEE, New York, 1997), Vol. 2, pp. 2651-2653.

[7] CST STUDIO SUITE 2018 (Dassault Systemes Deutschland $\mathrm{GmbH}$, Meitnerstr. 8, 70563 Stuttgart, Germany).

[8] B. Salvant, Impedance Model of the CERN SPS and Aspects of LHC Single-Bunch Stability, Ph.D. thesis, École polytechnique fédérale de Lausanne, 2010.

[9] T. Weiland, Electronics and communications, AEUE 31, 116 (1977).

[10] G. V. Stupakov, Wake and impedance, Report No. SLACPUB-8683, 2000.

[11] E. Gjonaj and T. Weiland, Impedance calculation, time domain, in Handbook of Accelerator Physics and Engineering, 2nd ed. (World Scientific Publishing, Singapore, 2013).

[12] K. Rothemund, H.-W. Glock, M. Borecky, and U. van Rienen, Eigenmode calculation in long and complex RF structures using the Coupled S-Parameter Calculation technique, DESY Report No. TESLA-REPORT 2000-33, 2000.

[13] K. Rothemund, H.-W. Glock, and U. van Rienen, Eigenmode calculation of complex RF-structures using S-parameters, IEEE Trans. Magn. 36, 1501 (2000).

[14] H.-W. Glock, K. Rothemund, and U. van Rienen, CSC-A procedure for Coupled S-Parameter Calculations, IEEE Trans. Magn. 38, 1173 (2002).

[15] D. Hecht, K. Rothemund, H.-W. Glock, and U. van Rienen, Computation of RF-properties of long and complex structures, in Proceedings of the 8th European Particle Accelerator Conference, Paris, 2002 (EPS-IGA and CERN, Geneva, 2002), pp. 1685-1687.
[16] K. Rothemund, Ein Verfahren zur Berechnung von Eigenmoden in langen und komplexen Hochfrequenzstrukturen, Ph.D. thesis, Universtät Rostock, 2004.

[17] T. Flisgen, H.-W. Glock, P. Zhang, I. R. R. Shinton, N. Baboi, R. M. Jones, and U. van Rienen, Scattering parameters of the $3.9 \mathrm{GHz}$ accelerating module in a free-electron laser linac: A rigorous comparison between simulations and measurements, Phys. Rev. Accel. Beams 17, 022003 (2014).

[18] T. Flisgen, H.-W. Glock, and U. van Rienen, A concatenation scheme for the computation of beam excited higher order mode port signals, in Proceedings of the 2nd International Particle Accelerator Conference, ICAP2011, San Sebastián, Spain (JACoW Publishing, Geneva, Switzerland, 2011), pp. 2238-2240.

[19] K. Y. Ng, Physics of Intensity Dependent Beam Instabilities (World Scientific, Singapore, 2006).

[20] M. Dohlus, R. Schuhmann, and T. Weiland, Calculation of frequency domain parameters using 3D eigensolutions, Int. J. Numer. Model. 12, 41 (1999).

[21] A. Tsakanian, T. Flisgen, H.-W. Glock, A. Velez, and J. Knobloch, HOM power levels in the BESSY VSR cold string, in Proceedings of 9th International Particle Accelerator Conference, ICAP2018, Vancouver, BC, Canada (JACoW Publishing, Geneva, Switzerland, 2018), pp. 2808-2811.

[22] S. G. Zadeh, U. van Rienen, T. Flisgen, and R. Calaga, HOM damping options for the Z-pole operating scenario of FCC-ee, in Proceedings of 10th International Particle Accelerator Conference, ICAP2019, Melbourne, Australia (JACoW Publishing, Geneva, Switzerland, 2019), pp. 590593.

[23] T. Weiland and R. Wanzenberg, Frontiers of particle beams: Intensity limitations: Proceedings of a topical course held by the Joint US-CERN School on Particle Accelerators, Hilton Head Island, South Carolina, 7-14 November 1990, Lect. Notes Phys. 400, 39 (1992).

[24] J. Schoberl and S. Zaglmayr, High order Nédélec elements with local complete sequence properties, COMPEL 24, 374 (2005).

[25] J. Jin, The Finite Element Method in Electromagnetics, 3rd ed. (Wiley-IEEE Press, New York, 2014).

[26] T. B. A. Senior, Impedance boundary conditions for imperfectly conducting surfaces, Appl. Sci. Res. 8, 418 (1960).

[27] B. Doliwa, H. D. Gersem, T. Weiland, and T. Boonen, Optimised electromagnetic $3 \mathrm{~d}$ field solver for frequencies below the first resonance, IET Sci. Meas. Technol. 57, 5356 (2007).

[28] U. van Rienen and T. Weiland, Impedances of cavities with beam ports above cut-off, in Proceedings of the 1986 International Linac Conference, Stanford, USA (1986), pp. 289-291, http://accelconf.web.cern.ch/AccelConf/186/ papers/we3-17.pdf.

[29] U. Niedermayer, Determination of beam coupling impedance in the frequency domain, Ph.D. thesis, Technische Universtät Darmstadt, 2015.

[30] E. Gjonaj and T. Weiland, A projection penalization approach for the high-order DG-FEM in the time domain, Radio Sci. 46, RS0E10 (2011). 
[31] E. Gjonaj, W. Ackermann, T. Lau, and T. Weiland, Coupler kicks in the third harmonic module for the XFEL, in Proceedings of the 23rd Particle Accelerator Conference, Vancouver, Canada, 2009 (JACoW Publication, Melbourne, Australia, 2009), pp. 1858-1860, http://accelconf.web.cern .ch/AccelConf/PAC2009/papers/we2pbc04.pdf.

[32] http://www.salome-platform.org/.

[33] MATLAB, Version R2017a (The MathWorks, Inc., Natick, Massachusetts, USA, 2017).

[34] See Supplemental Material at http://link.aps.org/ supplemental/10.1103/PhysRevAccelBeams.23.034601 scripts for concatenation and impedances as ASCII plain data.

[35] A. Henderson and J. Ahrens, The Paraview Guide: A Parallel Visualization Application (Kitware, Inc., New York, 2004).

[36] B. Aune et al., Superconducting TESLA cavities, Phys. Rev. Accel. Beams 3, 092001 (2000).

[37] R. Wanzenberg, Monopole, dipole and quadrupole passbands of the TESLA 9-cell Cavity, TESLA Report No. TESLA 2001-33. 